\title{
APPLICATION OF A NEW TYPE OF PREOPEN SETS AND RELATED CONTINUITY
}

\author{
BISHWAMBHAR ROY
}

\begin{abstract}
In this paper a new class of sets termed as $\tau_{\mu}$-preopen sets has been introduced and some of its properties are discussed. A new type of separation axiom has been introduced with the help of this newly defined sets. Finally some properties of weak forms of continuous functions have been studied.
\end{abstract}

\section{INTRODUCTION}

In 1982 Mashhour et al. [1] introduced a weaker form of open sets termed as preopen sets in topological spaces. Subsequently, Raychaudhury and Mukherjee introduced the class of $\delta$-preopen sets [2] containing the class of preopen sets with the help of $\delta$-open sets [3] introduced by Veličko. Recently, Altunoz and Aslim (2011) have introduced the concept of $\theta$-preopen sets [4] with the help of $\theta$-open sets [3] to study decomposition of continuity. My aim in this paper is to unify some results of these types of sets and some other similar types of sets with the help of a generalized topology [5] introduced by Á. Császár in 2002. Over the last few years, a large number of papers have been devoted to investigating different properties of generalized open sets. We recall some notions defined in [5]. Let $X$ be a non-empty set, $\mathcal{P}(X)$ denotes the power set of $X$. We call a class $\mu \subseteq \mathcal{P}(X)$ a generalized topology [5], (briefly, GT) if $\varnothing \in \mu$ and $\mu$ is closed under arbitrary union. A set $X$, with a GT $\mu$ on it is said to be a generalized topological space (briefly, GTS) and is denoted by $(X, \mu)$. The members of $\mu$ are called $\mu$-open sets and the complements of the $\mu$-open sets are called $\mu$-closed sets. For $A \subseteq X$, we denote by $c_{\mu}(A)$ the intersection of all $\mu$-closed sets containing $A$, i.e., the smallest $\mu$-closed set containing $A$; and by $i_{\mu}(A)$ the union of all $\mu$-open sets contained in $A$, i.e., the largest $\mu$-open set contained in $A[6,7]$. It is also well known from $[5,7,8]$ that if $\mu$ is a GT on $X$ and $A \subseteq X, x \in X$, then $x \in c_{\mu}(A)$ iff $x \in M \in \mu$ $\Rightarrow M \cap A \neq \varnothing$ and $c_{\mu}(X \backslash A)=X \backslash i_{\mu}(A)$.

Throughout the paper $(X, \tau)$ and $(Y, \sigma)$ will represent topological spaces and $\mu$ and $\lambda$ will be GT's on the topological space $(X, \tau)$ and $(Y, \sigma)$ respectively. $\operatorname{int}(A)$ and $\operatorname{cl}(A)$ will denote the interior and the closure of the set $A$, respectively.

$M S C$ (2010): primary 54A05, 54D20; secondary $54 \mathrm{C} 08$.

Keywords: $\tau_{\mu}$-preopen set, $\tau_{\mu}$-preclosed set, $D_{(\mu, p)}$-set, $\tau_{\mu}$-precontinuity.

The author acknowledges the financial support from UGC for the Major Research Project having grant No. 41-799/2012(SR) UGC, New Delhi. 


\section{2. $\tau_{\mu}$-PREOPEN SETS, $\tau_{\mu}$-PRECLOSED SETS AND THEIR PROPERTIES}

Definition 2.1. Let $\mu$ be a GT on a topological space $(X, \tau)$. A subset $A$ of $X$ is called $\tau_{\mu}$-preopen (or $\mu^{\prime}$-open [9]) if $A \subseteq \operatorname{int}\left(c_{\mu}(A)\right.$ ).

The complement of a $\tau_{\mu}$-preopen set is said to be a $\tau_{\mu}$-preclosed set, i.e., a subset $A$ of a topological space $(X, \tau)$ is said to be $\tau_{\mu}$-preclosed if $\operatorname{cl}\left(i_{\mu}(A)\right) \subseteq A$.

Remark 2.2. If in the above definition we put $\mu=\tau(\operatorname{resp} . \delta O(X), \mathcal{P}(X))$, then a $\tau_{\mu}$-preopen set reduces to a preopen [1] (resp. $\delta$-preopen [2], $\tau$-open) set.

Theorem 2.3. Let $\mu$ be a GT on a topological space $(X, \tau)$. If $A$ is open, then $A$ is $\tau_{\mu}$-preopen. Also if $A$ is $\tau_{\mu}$-preopen and $\mu$-closed, then $A$ is open.

Proof. If $A$ is open, then $A=\operatorname{int}(A) \subseteq \operatorname{int}\left(c_{\mu}(A)\right)$. Thus, $A$ is $\tau_{\mu}$-preopen.

Again, let $A$ be $\tau_{\mu}$-preopen and $\mu$-closed. Then, $A \subseteq \operatorname{int}\left(c_{\mu}(A)\right)=\operatorname{int}(A)$ showing $A$ to be open.

Example 2.4. Let $X=\{a, b, c\}, \tau=\{\varnothing,\{a\},\{b\},\{a, b\},\{a, c\}, X\}$ and $\mu=$ $\{\varnothing,\{a, b\},\{b, c\}, X\}$. Then, $\mu$ is a GT on the topological space $(X, \tau)$. It can be verified that $\{b, c\}$ is $\tau_{\mu}$-preopen but not open.

Theorem 2.5. Let $\mu$ be a GT on a topological space $(X, \tau)$. Then,

(a) arbitrary union of $\tau_{\mu}$-preopen sets is $\tau_{\mu}$-preopen i.e., the collection of all $\tau_{\mu}$-preopen sets is a $G T$ on $X$.

(b) arbitrary intersection of $\tau_{\mu}$-preclosed sets is $\tau_{\mu}$-preclosed.

Proof. (a) Let $\left\{A_{\alpha}: \alpha \in \Lambda\right\}$ be a family of $\tau_{\mu}$-preopen sets in $(X, \tau)$. Then, for each $\alpha \in \Lambda, A_{\alpha} \subseteq \operatorname{int}\left(c_{\mu}\left(A_{\alpha}\right)\right)$. Thus, $\cup\left\{A_{\alpha}: \alpha \in \Lambda\right\} \subseteq \cup\left\{\operatorname{int}\left(c_{\mu}\left(A_{\alpha}\right)\right): \alpha \in\right.$ $\Lambda\} \subseteq\left\{\operatorname{int}\left(c_{\mu}\left(\cup A_{\alpha}\right)\right): \alpha \in \Lambda\right\}$. Thus, $\cup\left\{A_{\alpha}: \alpha \in \Lambda\right\}$ is $\tau_{\mu}$-preopen.

(b) Follows from (a).

In general, the intersection of two $\tau_{\mu}$-preopen sets is not a $\tau_{\mu}$-preopen set as shown by the next example:

Example 2.6. Let $X=\{a, b, c\}, \tau=\{\varnothing,\{a\},\{a, b\},\{a, c\}, X\}$ and $\mu=$ $\{\varnothing,\{b\},\{a, b\},\{a, c\}, X\}$. Then, $\mu$ is a GT on the topological space $(X, \tau)$. It can be verified that $\{b, c\}$ and $\{a, c\}$ are two $\tau_{\mu}$-preopen sets but their intersection $\{c\}$ is not so.

Definition 2.7. Let $\mu$ be GT on a topological space $(X, \tau)$.

(a) The union of all $\tau_{\mu}$-preopen sets contained in $A$ is called the $\tau_{\mu}$-interior of $A$ and is denoted by $\tau_{\mu}-i n t(A)$.

(b) The intersection of all $\tau_{\mu}$-preclosed sets containing $A$ is called the $\tau_{\mu^{-}}$ closure of $A$ and is denoted by $\tau_{\mu}-c l(A)$.

Theorem 2.8. Let $\mu$ be a GT on a topological space $(X, \tau)$. Then, for any two subsets $A$ and $B$ of $X$,

(i) $\tau_{\mu}-c l(X)=X$ and $\tau_{\mu}-c l(\varnothing)=\varnothing$.

(ii) $A \subseteq \tau_{\mu}-\operatorname{cl}(A)$.

(iii) $A \subseteq B \Rightarrow \tau_{\mu}-c l(A) \subseteq \tau_{\mu}-c l(B)$.

(iv) $\tau_{\mu}-\operatorname{cl}(A) \cup \tau_{\mu}-c l(B) \subseteq \tau_{\mu}-c l(A \cup B)$. 
(v) $\tau_{\mu}-c l(A \cap B) \subseteq \tau_{\mu}-\operatorname{cl}(A) \cap \tau_{\mu}-\operatorname{cl}(B)$.

(vi) $A$ is $\tau_{\mu}$-preclosed if and only if $A=\tau_{\mu}-c l(A)$.

(vii) $x \in \tau_{\mu}-\operatorname{cl}(A)$ if and only if, for each $\tau_{\mu}$-preopen set $V$ containing $x, V \cap A \neq$ $\varnothing$.

(viii) $\tau_{\mu}-c l\left(\tau_{\mu}-c l(A)\right)=\tau_{\mu}-c l(A)$.

Proof. We shall prove (viii) only.

To prove (viii), it is sufficient to show that $\tau_{\mu}-c l\left(\tau_{\mu}-c l(A)\right) \subseteq \tau_{\mu^{-}}-c l(A)$ and the rest follows from (ii). Let $x \notin \tau_{\mu}$-cl $(A)$. Then, by (vii), there exists a $\tau_{\mu}$-preopen set $V$ containing $x$ such that $V \cap A=\varnothing$. Then, $A \subseteq X \backslash V$ where $X \backslash V$ is a $\tau_{\mu^{-}}$ preclosed set. Then, by (iii) and (vi), $\tau_{\mu}-\operatorname{cl}\left(\tau_{\mu}-c l(A)\right) \subseteq \tau_{\mu}-\operatorname{cl}\left(\tau_{\mu}-c l(X \backslash V)\right)=X \backslash V$. Thus, $x \notin \tau_{\mu}-\operatorname{cl}\left(\tau_{\mu}-c l(A)\right)$.

Example 2.9. (a) Let $X=\{a, b, c\}, \tau=\{\varnothing,\{b\},\{a, b\}, X\}$ and $\mu=\{\varnothing,\{a, b\}$, $\{b, c\}, X\}$. Then, $\mu$ is a GT on the topological space $(X, \tau)$. It can be verified easily that $\tau_{\mu}-c l(\{b, c\}) \cap \tau_{\mu}-c l(\{a, c\}) \neq \tau_{\mu}-c l(\{c\})$.

(b) Let $X=\{a, b, c\}, \tau=\{\varnothing,\{b\},\{a, b\},\{b, c\}, X\}$ and $\mu=\{\varnothing,\{b\},\{c\},\{a, b\}$, $\{a, c\},\{b, c\}, X\}$. Then, $\mu$ is a $\mathrm{GT}$ on the topological space $(X, \tau)$. It can be verified that $\{b\} \subseteq\{b, c\}$ but $\tau_{\mu}-c l(\{b\})=\tau_{\mu}-c l(\{b, c\})$.

(c) Let $X=\{a, b, c\}, \tau=\{\varnothing,\{a\},\{a, b\},\{a, c\}, X\}$ and $\mu=\{\varnothing,\{a, b\},\{b, c\}$, $\{a, c\}, X\}$. Then, $\mu$ is a GT on the topological space $(X, \tau)$. It can be verified easily that $\tau_{\mu}-c l(\{a\}) \cup \tau_{\mu}-c l(\{b\}) \neq \tau_{\mu}-c l(\{a, b\})$.

Theorem 2.10. Let $\mu$ be a GT on a topological space $(X, \tau)$. Then, for any two subsets $A$ and $B$ of $X$,

(i) $\tau_{\mu}-\operatorname{int}(X)=X$ and $\tau_{\mu}-\operatorname{int}(\varnothing)=\varnothing$.

(ii) $\tau_{\mu}-$ int $(A) \subseteq A$.

(iii) $A \subseteq B \Rightarrow \tau_{\mu}-\operatorname{int}(A) \subseteq \tau_{\mu}-\operatorname{int}(B)$.

(iv) $\tau_{\mu}-\operatorname{int}(A) \cup \tau_{\mu}-i n t(B) \subseteq \tau_{\mu}-i n t(A \cup B)$.

(v) $\tau_{\mu}-i n t(A \cap B) \subseteq \tau_{\mu}-i n t(A) \cap \tau_{\mu}-i n t(B)$.

(vi) $A$ is $\tau_{\mu}$-preopen if and only if $A=\tau_{\mu}$-int $(A)$.

(vii) $x \in \tau_{\mu}$-int $(A)$ if and only if there exists a $\tau_{\mu}$-preopen set $V$ containing $x$ such that $V \subseteq A$.

(viii) $\tau_{\mu}-\operatorname{int}\left(\tau_{\mu}-\operatorname{int}(A)\right)=\tau_{\mu}-\operatorname{int}(A)$.

Proof. Obvious.

Theorem 2.11. Let $\mu$ be a GT on a topological space $(X, \tau)$. Then, the following equalities are true:

(i) $\tau_{\mu}-\operatorname{cl}(X \backslash A)=X \backslash \tau_{\mu}-\operatorname{int}(A)$.

(ii) $\tau_{\mu}-i n t(X \backslash A)=X \backslash \tau_{\mu}-c l(A)$.

Proof. Follows from the fact that $A$ is a $\tau_{\mu}$-preopen set if and only if $X \backslash A$ is a $\tau_{\mu}$-preclosed set.

\section{3. $D_{(\mu, p)}$-SETS AND Their ASSOCIATED PROPERTIES}

Definition 3.1. Let $\mu$ be a GT on a topological space $(X, \tau)$. A subset $A$ of $X$ is called a $D_{(\mu, p)}$-set if there exist two $\tau_{\mu}$-preopen sets $G$ and $H$ with $G \neq X$ and $A=G \backslash H$. 
It follows from the above definition that every $\tau_{\mu}$-preopen set $A$ except $X$ is a $D_{(\mu, p)}$-set.

Example 3.2. Let $X=\{a, b, c\}, \tau=\{\varnothing,\{a\},\{a, b\},\{a, c\}, X\}$ and $\mu=$ $\{\varnothing,\{b\},\{a, b\},\{a, c\}, X\}$. Then, $\mu$ is a GT on the topological space $(X, \tau)$. It can be verified that the collection of all $\tau_{\mu}$-preopen sets is $\{\varnothing,\{a\},\{a, b\},\{a, c\},\{b, c\}$, $X\}$. Thus, $\{c\}$ is a $D_{(\mu, p)}$-set which is not a $\tau_{\mu}$-preopen set.

Definition 3.3. Let $\mu$ be a GT on a topological space $(X, \tau)$. Then, $(X, \tau)$ is said to be $(\mu, p)-D_{1}$ if, for any two points $x, y \in X$ with $x \neq y$, there exist two $D_{(\mu, p)}$-sets $U$ and $V$ of $X$ such that $x \in U, y \notin U$ and $y \in V, x \notin V$.

Definition 3.4. Let $\mu$ be a GT on a topological space $(X, \tau)$. Then, $(X, \tau)$ is said to be

(i) $\tau_{\mu}-T_{0}$ if, for any two distinct points $x, y$ of $X$ there exists a $\tau_{\mu}$-preopen set containing $x$ but not $y$ or a $\tau_{\mu}$-preopen set containing $y$ but not $x$;

(ii) $\tau_{\mu}-T_{1}$ if, for any two distinct points $x, y$ of $X$ there exist a $\tau_{\mu}$-preopen set of $X$ containing $x$ but not $y$ and a $\tau_{\mu}$-preopen set of $X$ containing $y$ but not $x$;

(iii) $\tau_{\mu}-T_{2}$ if, for any two distinct points $x, y$ of $X$ there exist two disjoint $\tau_{\mu}$-preopen sets $G$ and $H$ of $X$ containing $x$ and $y$ respectively.

Remark 3.5. Let $\mu$ be a GT on a topological space $(X, \tau)$. Then,

(i) every $\tau_{\mu}-T_{i}$ space is a $\tau_{\mu}-T_{i-1}$ space, for $i=1,2$.

(ii) every $\tau_{\mu}-T_{1}$ space is a $(\mu, p)-D_{1}$ space.

Theorem 3.6. Let $\mu$ be a GT on a topological space $(X, \tau)$. Then, $(X, \tau)$ is $a(\mu, p)-D_{1}$ space if and only if, for any two distinct points $x, y$ of $X$, there exist two disjoint $D_{(\mu, p)}$-sets $G$ and $H$ of $X$ containing $x$ and $y$ respectively.

Proof. One part of the theorem follows from definition of a $(\mu, p)-D_{1}$ space.

Conversely, assume that $X$ is a $(\mu, p)-D_{1}$ space and $x, y$ are two distinct points of $X$. Then, there exist two $D_{(\mu, p)}$-sets $G_{1}$ and $G_{2}$ such that $x \in G_{1}, y \notin G_{1}$; $y \in G_{2}, x \notin G_{2}$. Let $G_{1}=U_{1} \backslash U_{2}$ and $G_{2}=V_{1} \backslash V_{2}$. From $x \notin G_{2}$, we have either $x \notin V_{1}$ or $x \in V_{1} \cap V_{2}$.

Case 1: $x \notin V_{1}$. From $y \notin G_{1}$, we have the following two subcases: (a) $y \notin U_{1}$. From $x \in U_{1} \backslash U_{2}$ we have $x \in U_{1} \backslash\left(U_{2} \cup V_{1}\right)$ and, from $y \in V_{1} \backslash V_{2}$, we have $y \in V_{1} \backslash\left(U_{1} \cup V_{2}\right)$. It can be verified that $U_{1} \backslash\left(U_{2} \cup V_{1}\right)$ and $V_{1} \backslash\left(U_{1} \cup V_{2}\right)$ are disjoint.

(b) $y \in U_{1} \cap U_{2}$. We have $x \in U_{1} \backslash U_{2}, y \in U_{2}$ and $\left(U_{1} \backslash U_{2}\right) \cap U_{2}=\varnothing$.

Case 2: $x \in V_{1} \cap V_{2}$ implies that $x \in V_{1}$ and $x \in V_{2}$. We have $y \in V_{1} \backslash V_{2}$, $x \in V_{2}$ and $\left(V_{1} \backslash V_{2}\right) \cap V_{2}=\varnothing$.

Example 3.7. (i) Consider Example 3.2. It is easy to observe that $(X, \tau)$ is a $\tau_{\mu}-T_{1}$ space but not a $\tau_{\mu}-T_{2}$ space.

(ii) Let $X=\{a, b, c\}, \tau=\{\varnothing,\{b\},\{a, b\},\{b, c\}, X\}$ and $\mu=\mathcal{P}(X)$. Then, $\mu$ is a GT on the topological space $(X, \tau)$. It can be verified that $(X, \tau)$ is not a $\tau_{\mu}-T_{1}$ space. It can also be verified that $(X, \tau)$ is a $(\mu, p)-D_{1}$ space.

(iii) let $X=\{a, b, c\}, \tau=\{\varnothing,\{b\},\{a, b\}, X\}$ and $\mu=\{\varnothing,\{a\},\{a, b\},\{a, c\}$, $\{b, c\}, X\}$. Then, $\mu$ is a GT on the topological space $(X, \tau)$. It can be verified that $(X, \tau)$ is a $\tau_{\mu}-T_{0}$ space but not a $(\mu, p)-D_{1}$ space. 
Theorem 3.8. Let $\mu$ be a GT on a topological space. Then, $(X, \mu)$ is $\tau_{\mu}-T_{0}$ if and only if, for any two distinct points $x, y$ of $X$, there exists a $D_{(\mu, p)}$-set of $X$ containing $x$ but not $y$ or a $D_{(\mu, p)}$-set of $X$ containing $y$ but not $x$.

Proof. If $(X, \mu)$ is $\tau_{\mu}-T_{0}$, then the condition holds.

Conversely, let $x$ and $y$ be two distinct points of $X$. Without loss of generality we may assume that there exists a $D_{(\mu, p)}$-set $U$ of $X$ containing $x$ but not $y$. So there exist two $\tau_{\mu}$-preopen sets $G$ and $H$ with $U \neq X$ and $U=G \backslash H$. Hence, $x \in G \backslash H$ and $y \notin G \backslash H$. Thus, $x \in G$ and $x \notin H$.

Case 1: $y \notin G$. Then, the proof is complete.

Case 2: $y \in G$. If $y \in G$, then $y \in H$ and $x \notin H$. Thus, $(X, \tau)$ is $\tau_{\mu}-T_{0}$.

Remark 3.9. In view of Remark 3.5, we have the following diagram.

$$
\begin{array}{ccc}
\tau_{\mu}-T_{2} \Rightarrow & \tau_{\mu}-T_{1} \Rightarrow \quad \tau_{\mu}-T_{0} \\
\Downarrow & \\
& \\
& & \\
& & \\
& &
\end{array}
$$

Definition 3.10. Let $\mu$ be a GT on a topological space $(X, \tau)$. A point $x \in X$ is said to be a $(\mu, p)$-neat point if there does not exist any $\tau_{\mu}$-preopen set containing $x$ other than $X$.

Theorem 3.11. Let $\mu$ be a GT on a topological space $(X, \tau)$ which is $\tau_{\mu}-T_{0}$. Then, $(X, \tau)$ is a $(\mu, p)-D_{1}$ space if and only if $(X, \tau)$ has no $(\mu, p)$-neat point.

Proof. Suppose that $(X, \tau)$ is a $(\mu, p)-D_{1}$ space. Then, each $x \in X$ is contained in a $D_{(\mu, p)}$-set $O(=U \backslash V)$ of $X$. By the definition of a $D_{(\mu, p)}$-set, $U \neq X$ and $U$ is $\tau_{\mu}$-preopen. Thus, $x$ is not a $(\mu, p)$-neat point.

Conversely, since $(X, \tau)$ is $\tau_{\mu}-T_{0}$ for each pair of distinct points $x, y \in X$, at least one of them, choose $x$ for example, is contained in a $\tau_{\mu}$-preopen set $U$ not containing the point $y$. Since, by the hypothesis, $X$ has no $(\mu, p)$-neat point, $y$ is not a $(\mu, p)$-neat point. So there exists a $\tau_{\mu}$-preopen set $V(\neq X)$ containing $y$. Thus, $y \in V \backslash U$ and $x \notin V \backslash U$ which is a $D_{(\mu, p)}$-set.

Definition 3.12. Let $\mu$ and $\lambda$ be two GTs on the topological spaces $(X, \tau)$ and $(Y, \sigma)$, respectively. A function $f:(X, \tau) \rightarrow(Y, \sigma)$ is said to be $\left(\tau_{\mu}, \sigma_{\lambda}\right)$ precontinuous if $f^{-1}(V)$ is $\tau_{\mu}$-preopen for each $\sigma_{\lambda}$-preopen set $V$ of $Y$.

Theorem 3.13. Let $\mu$ and $\lambda$ be two GTs on the topological spaces $(X, \tau)$ and $(Y, \sigma)$, respectively. If the function $f:(X, \tau) \rightarrow(Y, \sigma)$ is $\left(\tau_{\mu}, \sigma_{\lambda}\right)$-precontinuous and surjective, then the inverse image of a $D_{(\lambda, p)}$-set in $(Y, \sigma)$ is a $D_{(\mu, p)}$-set in $(X, \tau)$.

Proof. Let $F$ be a $D_{(\lambda, p)}$-set in $Y$. Then, there exist two $\sigma_{\lambda}$-preopen sets $U$ and $V$ with $U \neq Y$ and $F=U \backslash V$. By the $\left(\tau_{\mu}, \sigma_{\lambda}\right)$-precontinuity of $f, f^{-1}(U)$ and $f^{-1}(V)$ are $\tau_{\mu}$-preopen in $X$. Clearly, $f^{-1}(U) \neq X$ and $f^{-1}(F)=f^{-1}(U) \backslash f^{-1}(V)$ showing $f^{-1}(F)$ to be a $D_{(\mu, p)}$-set.

Theorem 3.14. Let $\mu$ and $\lambda$ be two GTs on the topological spaces $(X, \tau)$ and $(Y, \sigma)$, respectively. If the function $f:(X, \tau) \rightarrow(Y, \sigma)$ is $\left(\tau_{\mu}, \sigma_{\lambda}\right)$-precontinuous and bijective and $(Y, \sigma)$ is $(\lambda, p)-D_{1}$, then $(X, \tau)$ is $(\mu, p)-D_{1}$. 
Proof. Let $(Y, \sigma)$ be a $(\lambda, p)-D_{1}$ space and $x, y$ be two distinct points of $X$. Then, by injectiveness of $X, f(x), f(y) \in Y$ with $f(x) \neq f(y)$. Thus, there exist two $D_{(\lambda, p)}$-sets $U$ and $V$ containing $f(x)$ and $f(y)$ respectively such that $f(x) \notin V$ and $f(y) \notin U$. Then, $f^{-1}(U)$ and $f^{-1}(V)$ are two $D_{(\mu, p)}$-sets (by Theorem 3.13) containing $x$ and $y$ respectively such that $y \notin f^{-1}(U)$ and $x \notin f^{-1}(V)$. Thus, $(X, \tau)$ is a $(\mu, p)-D_{1}$ space.

Theorem 3.15. Let $\mu$ be a GT on the topological space $(X, \tau)$. Then, $(X, \tau)$ is $(\mu, p)-D_{1}$ if and only if, for any $x \neq y$ in $X$, there exists a topological space $(Y, \sigma)$ which is a $(\lambda, p)-D_{1}$ space for some $G T \lambda$ on the topological space $(Y, \sigma)$ and there exists a surjective $\left(\tau_{\mu}, \sigma_{\lambda}\right)$-precontinuous function $f:(X, \tau) \rightarrow(Y, \sigma)$ with $f(x) \neq f(y)$.

Proof. One part of the theorem is trivial as for every pair of distinct points of $X$, it is sufficient to take the identity function on $X$.

Conversely, let $x, y$ be two distinct points of $X$. Then, by the hypothesis, there exists a $\left(\tau_{\mu}, \sigma_{\lambda}\right)$-precontinuous function $f:(X, \tau) \rightarrow(Y, \sigma)$ such that $f(x) \neq f(y)$ where $(Y, \sigma)$ is a $(\lambda, p)-D_{1}$ space. Then, there exist two disjoint $D_{(\lambda, p)}$-sets $U$ and $V$ containing $f(x)$ and $f(y)$ respectively (by Theorem 3.6). Thus, by Theorem $3.13 f^{-1}(U)$ and $f^{-1}(V)$ are two $D_{(\mu, p)}$-sets containing $x$ and $y$ respectively. The rest follows from Theorem 3.6.

\section{4. $\tau_{\mu}$-PRECONTINUOUS FUNCTION AND THEIR ASSOCIATED PROPERTIES}

Definition 4.1. A function $f:(X, \tau) \rightarrow(Y, \sigma)$ is said to be $\tau_{\mu}$-precontinuous if, for each open set $V$ of $Y, f^{-1}(V)$ is $\tau_{\mu}$-preopen.

It follows from the above definition that every continuous function is $\tau_{\mu}$-precontinuous and every $\left(\tau_{\mu}, \sigma_{\lambda}\right)$-precontinuous function is $\tau_{\mu}$-precontinuous but the converses are false as shown by the next examples.

Example 4.2. (i) Let $X=Y=\{a, b, c\}, \tau=\{\varnothing,\{a\},\{a, b\},\{a, c\}, X\}$, $\sigma=\{\varnothing,\{c\},\{a, b\}, Y\}$ and $\mu=\{\varnothing,\{a, b\},\{a, c\}, X\}$. Then, $\mu$ is a GT on the topological space $(X, \tau)$. Consider the mapping $f:(X, \tau) \rightarrow(Y, \sigma)$ defined by $f(a)=c ; f(b)=a$ and $f(c)=b$. It is easy to see that $f$ is $\tau_{\mu}$-precontinuous but not continuous.

(ii) Let $X=Y=\{a, b, c\}, \tau=\sigma=\{\varnothing,\{a\},\{a, b\}, X\}, \mu=\mathcal{P}(X)$, the power set of $X$ and $\lambda=\{\varnothing,\{b\},\{c\},\{b, c\},\{a, b\},\{a, c\}, X\}$. Then, $\mu$ and $\lambda$ are GTs on the topological spaces $(X, \tau)$ and $(Y, \sigma)$ respectively. Consider the identity mapping $f:(X, \tau) \rightarrow(Y, \sigma)$. It is easy to see that $f$ is $\tau_{\mu}$-precontinuous but not $\left(\tau_{\mu}, \sigma_{\lambda}\right)$-precontinuous.

Definition 4.3. A function $f:(X, \tau) \rightarrow(Y, \sigma)$ is said to be contra $\mu$-continuous [9] if $f^{-1}(V)$ is $\mu$-closed in $X$ for each open set $V$ in $Y$.

Theorem 4.4. If $f:(X, \tau) \rightarrow(Y, \sigma)$ is contra $\mu$-continuous then $f$ is continuous if and only if it is $\tau_{\mu}$-precontinuous.

Proof. One part of the theorem is trivial as every continuous function is $\tau_{\mu^{-}}$ precontinuous. 
Conversely, let $f$ be $\tau_{\mu}$-precontinuous and let $V$ be an open set in $Y$. Then, $f^{-1}(V)$ is $\mu$-closed and $\tau_{\mu}$-preopen (by Definition 4.1 and 4.3). Thus, by Theorem $2.3, f^{-1}(V)$ is open and hence $f$ is a continuous function.

Theorem 4.5. Let $f:(X, \tau) \rightarrow(Y, \sigma)$ be a function. Then, the following statements are equivalent:

(i) $f$ is $\tau_{\mu}$-precontinuous;

(ii) for each $x \in X$ and each $V \in \sigma$ containing $f(x)$, there exists a $\tau_{\mu}$-preopen set $U$ in $X$ containing $x$ such that $f(U) \subseteq V$ that is, $f$ is $\tau_{\mu}$-precontinuous at each point $x \in X$;

(iii) inverse image of every closed set in $Y$ is $\tau_{\mu}$-preclosed in $X$;

(iv) $\tau_{\mu}-c l\left(f^{-1}(B)\right) \subseteq f^{-1}(\operatorname{cl}(B))$ for every $B \subseteq Y$;

(v) $f\left(\tau_{\mu}-c l(A)\right) \subseteq \operatorname{cl}(f(A))$, for each $A \subseteq X$;

(vi) $f^{-1}(\operatorname{int}(B)) \subseteq \tau_{\mu}-\operatorname{int}\left(f^{-1}(B)\right)$ for any $B \subseteq Y$.

Proof. (i) $\Leftrightarrow$ (ii): Let $x \in X$ and $V$ be an open set in $Y$ containing $f(x)$. Then, by (i), $f^{-1}(V)$ is $\tau_{\mu}$-preopen. Let $U=f^{-1}(V)$. Then, $x \in U$ and $f(U) \subseteq V$.

Conversely, suppose that $x \in X$ and $V \in \sigma$ contains $f(x)$. Then, by (ii), there exists a $\tau_{\mu}$-preopen set $U_{x}$ in $X$ containing $x$ such that $f\left(U_{x}\right) \subseteq V$ i.e., $U_{x} \subseteq f^{-1}(V)$. Thus, $f^{-1}(V)=\cup\left\{U_{x}: x \in f^{-1}(V)\right\}$. Thus, by Theorem 2.5, $f^{-1}(V)$ is $\tau_{\mu}$-preopen.

(i) $\Leftrightarrow$ (iii): Obvious.

(iii) $\Rightarrow$ (iv) : Let $B \subseteq Y$ and $f^{-1}(c l(B))$ is $\tau_{\mu}$-preclosed in $X$. Then, $\tau_{\mu^{-}}$ $c l\left(f^{-1}(B)\right) \subseteq \tau_{\mu}-c l\left(f^{-1}(\operatorname{cl}(B))\right)=f^{-1}(\operatorname{cl}(B))$.

(iv) $\Rightarrow(\mathrm{v})$ : Let $A \subseteq X$. Then, $f(A) \subseteq Y$. Thus, by (iv), we have $\tau_{\mu^{-}} c l(A) \subseteq \tau_{\mu^{-}}$ $c l\left(f^{-1}(f(A))\right) \subseteq f^{-1}(c l(f(A)))$. Thus, $f\left(\tau_{\mu}-c l(A)\right) \subseteq \operatorname{cl}(f(A))$.

$(\mathrm{v}) \Rightarrow$ (iii): Let $F$ be a closed set in $Y$. Then, by $(\mathrm{v}), f\left(\tau_{\mu}-c l\left(f^{-1}(F)\right)\right) \subseteq$ $c l\left(f\left(f^{-1}(F)\right)\right) \subseteq \operatorname{cl}(F)=F$. Thus, $\tau_{\mu^{-}} c l\left(f^{-1}(F)\right) \subseteq f^{-1}(F)$ showing $f^{-1}(F)$ to be $\tau_{\mu}$-preclosed.

(i) $\Rightarrow\left(\right.$ vi): Let $B$ be any subset of $Y$. Then, $f^{-1}(\operatorname{int}(B))$ is a $\tau_{\mu}$-preopen set in $X$. Hence $f^{-1}(\operatorname{int}(B))=\tau_{\mu}-\operatorname{int}\left(f^{-1}(\operatorname{int}(B))\right) \subseteq \tau_{\mu}-\operatorname{int}\left(f^{-1}(B)\right)$.

$($ vi $) \Rightarrow(\mathrm{i})$ : Let $U$ be an open set in $Y$. Then, $f^{-1}(U)=f^{-1}(\operatorname{int}(U)) \subseteq \tau_{\mu^{-}}$ $\operatorname{int}\left(f^{-1}(U)\right)$. Hence $f^{-1}(U)$ is $\tau_{\mu}$-preopen.

Theorem 4.6. If $f:(X, \tau) \rightarrow(Y, \sigma)$ is $\tau_{\mu}$-precontinuous and $g:(Y, \sigma) \rightarrow(Z, \delta)$ is continuous, then $g_{\circ} f:(X, \tau) \rightarrow(Z, \delta)$ is $\tau_{\mu}$-precontinuous.

Proof. Obvious.

Theorem 4.7. If $f:(X, \tau) \rightarrow(Y, \sigma)$ is a $\tau_{\mu}$-precontinuous injection and $(Y, \sigma)$ is $T_{i}$, then $(X, \tau)$ is $\tau_{\mu}-T_{i}$, where $i=0,1,2$.

Proof. We prove the theorem for $i=2$. Let $Y$ be a $T_{2}$ space and $x, y$ be two distinct points in $X$. Since $f$ is injective, $f(x)$ and $f(y)$ are distinct points of $Y$. Then, there exist two disjoint open sets $G$ and $H$ containing $f(x)$ and $f(y)$ respectively. Thus, by $\tau_{\mu}$-precontinuity, $f^{-1}(G)$ and $f^{-1}(H)$ are $\tau_{\mu}$-preopen sets containing $x$ and $y$ respectively. Also, $f^{-1}(G)$ and $f^{-1}(H)$ are disjoint. Thus, $(X, \tau)$ is a $\tau_{\mu}-T_{2}$ space. 
Definition 4.8. A function $f:(X, \tau) \rightarrow(Y, \sigma)$ is said to have a $\left(\tau_{\mu}, \tau\right)$-graph $G(f)$ if, for each $(x, y) \in(X \times Y) \backslash G(f)$, there exist a $\tau_{\mu}$-preopen set $U$ containing $x$ in $X$ and an open set $V$ of $Y$ containing $y$ such that $(U \times V) \cap G(f)=\varnothing$.

Lemma 4.9. A function $f:(X, \tau) \rightarrow(Y, \sigma)$ has a $\left(\tau_{\mu}, \tau\right)$-graph if and only if, for each $(x, y) \in X \times Y$ with $y \neq f(x)$, there exist a $\tau_{\mu}$-preopen set $U$ and an open set $V$ containing $x$ and $y$ respectively, such that $f(U) \cap V=\varnothing$.

Proof. Follows from Definition 4.8.

Theorem 4.10. If $f:(X, \tau) \rightarrow(Y, \sigma)$ is a $\tau_{\mu}$-precontinuous function and $(Y, \sigma)$ is Hausdorff, then $f$ has a $\left(\tau_{\mu}, \tau\right)$-graph.

Proof. Let $(x, y) \in X \times Y$ be such that $f(x) \neq y$. Then, by the Hausdorffness of $Y$, there exist disjoint open sets $G$ and $H$ containing $y$ and $f(x)$ respectively. So, there exists a $\tau_{\mu}$-preopen set $U$ containing $x$ such that $f(U) \subseteq H$. Thus, $f(U) \cap G \subseteq H \cap G=\varnothing$. Thus, $f$ has a $\left(\tau_{\mu}, \tau\right)$-graph.

Definition 4.11. Let $\mu$ be a $\mathrm{GT}$ on a topological space $(X, \tau)$. Then, a subset $A$ of $X$ is said to be $\tau_{\mu}$-compact if every cover of $A$ by $\tau_{\mu}$-preopen sets of $X$ has a finite subcover.

Theorem 4.12. If $f:(X, \tau) \rightarrow(Y, \sigma)$ has a $\left(\tau_{\mu}, \tau\right)$-graph, then $f(K)$ is closed in $(Y, \sigma)$, for each subset $K$ which is $\tau_{\mu}$-compact relative to $(X, \tau)$.

Proof. Let $y \notin f(K)$. Then, for each $x \in K,(x, y) \notin G(f)$. Since $f$ has a $\left(\tau_{\mu}, \tau\right)$-graph, there exist a $\tau_{\mu}$-preopen set $G_{x}$ containing $x$ and an open set $H_{x}$ containing $y$ such that $f\left(G_{x}\right) \cap H_{x}=\varnothing$. Then, the family $\left\{G_{x}: x \in K\right\}$ is a cover of $K$ by $\tau_{\mu}$-preopen sets of $X$. Thus, there exists a finite subset $K_{0}$ of $K$ such that $K \subseteq \cup\left\{G_{x}: x \in K_{0}\right\}$. Let $H=\cap\left\{H_{x}: x \in K_{0}\right\}$. Then, $H$ is an open set containing $y$. Also, $f(K) \cap H \subseteq \cup\left\{f\left(G_{x}\right): x \in K_{0}\right\} \cap H \subseteq \cup\left\{f\left(G_{x}\right) \cap H: x \in\right.$ $\left.K_{0}\right\}=\varnothing$. Thus, $y \notin c l(f(K))$. Therefore, $f(K)$ is closed in $(Y, \sigma)$.

Definition 4.13. Let $\mu$ be a GT on a topological space $(X, \tau)$. Then, the $\tau_{\mu}$-frontier (see [10]) of a subset $A$ of $X$ is denoted by $\tau_{\mu}-F r(A)$ and is defined by $\tau_{\mu}-\operatorname{Fr}(A)=\tau_{\mu}-\operatorname{cl}(A) \cap \tau_{\mu}-\operatorname{cl}(X \backslash A)$.

Theorem 4.14. For a function $f:(X, \tau) \rightarrow(Y, \sigma)$ the following statements are equivalent:

(a) $f$ is not $\tau_{\mu}$-precontinuous at $x$,

(b) $x \in \cup\left\{\tau_{\mu}-F r\left(f^{-1}(V)\right): V \in \sigma, f(x) \in V\right\}$,

(c) there exists an open set $V$ of $Y$ containing $f(x)$ with $x \in \tau_{\mu}-F r\left(f^{-1}(V)\right)$.

Proof. $(a) \Rightarrow(b)$ : Let us assume that $f$ is not $\tau_{\mu}$-precontinuous at $x \in X$. Then, there exists an open set $V$ of $Y$ containing $f(x)$ such that, for every $\tau_{\mu}$ preopen set $U$ containing $x, U \cap\left(X \backslash f^{-1}(V)\right) \neq \varnothing$. Thus, $x \in \tau_{\mu}-c l\left(X \backslash f^{-1}(V)\right)$. Also, $x \in f^{-1}(V) \subseteq \tau_{\mu^{-}} c l\left(f^{-1}(V)\right)$. Thus, $x \in \tau_{\mu^{-}} \operatorname{Fr}\left(f^{-1}(V)\right)$ Thus, $x \in \tau_{\mu^{-}}$ $\operatorname{Fr}\left(f^{-1}(V)\right)$ where $V$ is an open set in $Y$ containing $f(x)$.

$(b) \Rightarrow(c)$ : Obvious.

$(c) \Rightarrow(a)$ : Assume that $f$ is $\tau_{\mu}$-precontinuous at $x \in X$ and $V$ is any open set of $Y$ containing $f(x)$. Then, there exists a $\tau_{\mu}$-preopen set $U$ containing $x$ such that $f(U) \subseteq V$. Thus, $x \subseteq U \subseteq f^{-1}(V)$ and hence $x \in \tau_{\mu^{-}} i n t\left(f^{-1}(V)\right) \subseteq X \backslash \tau_{\mu^{-}}$ $\operatorname{Fr}\left(f^{-1}(V)\right)$. Thus, $x \notin \tau_{\mu}-F r\left(f^{-1}(V)\right)$. 


\section{Conclusion}

The new class of subsets introduced in this paper can be applied in different fields of mathematics such as rough set theory [11] and granular computing [12]. On the other hand, maps are always important in all branches of mathematics. This is why generalized open sets and generalized continuous functions are currently among the most interesting areas of research.

Acknowledgement. The author is thankful to the referees for the valuable comments to improve the paper.

\section{REFERENCES}

[1] A. S. Mashhour, M. E. Abd.El-Monsef and S. N. El-Deep, On precontinuous mappings, Proc. Math. Phys. Soc. Egypt 53 (1982), 47-53.

[2] S. Raychaudhuri and M. N. Mukherjee, On $\delta$-almost continuity and $\delta$-preopen sets, Bull. Inst. Math., Acad. Sinica 21 (1993), 357-366.

[3] N. V. Veličko, H-closed topological spaces, Mat. Sb. 70 (1966), 98-112.

[4] T. Altunöz and G. Aslim, On new decomposition of continuity, J. Adv. Res. Pure Math. 3 (2011), 84-92.

[5] Á. Császár, Generalized topology, generalized continuity, Acta Math. Hungar. 96 (2002), 351-357.

[6] Á. Császár, Generalized open sets in generalized topologies, Acta Math. Hungar. 106 (2005), 53-66.

[7] Á. Császár, Remarks on quasi topologies, Acta Math. Hungar. 119 (2008), 197-200.

[8] B. Roy, A note on weakly $(\mu, \lambda)$-closed function, Math. Bohemica 138 (2013), 397-405.

[9] B. Roy and R. Sen, On a type of decomposition of continuity, Afrika Math. 26 (2015), 153-158.

[10] B. Roy, On weakly ( $\mu, \lambda)$-open functions, Ukrainian Math. Jour. 66 (2015), 1595-1602.

[11] A. Wiweger, On topological rough sets, Bull. Pol. Acad. Sci., Math. 37 (1989), 89-93.

[12] R. Bello, R. Falcon, W. Pedrycz and J. Kacprzyk (eds.), Granular Computing: At the Junction of Rough Sets and Fuzzy Sets, Studies in Fuzziness and Soft Computing 224, Springer-Verlag, Berlin, Heidelberg, 2008.

Bishwambhar Roy, Department of Mathematics, Women's Christian College, 6, Greek Church Row, Kolkata-700 026, India

e-mail: bishwambhar_roy@yahoo.co.in 
